\title{
EnCal: An Automated Forward Deduction System for General-Purpose Entailment Calculus
}

\author{
Jingde Cheng \\ Department of Computer Science and Communication Engineering \\ Kyushu University \\ 6-10-1 Hakozaki, Fukuoka 812-81, Japan \\ cheng@csce.kyushu-u.ac.jp
}

\begin{abstract}
This paper presents the fundamental design ideas, working principles, and implementation of an automated forward deduction system for general-purpose entailment calculus, named EnCal, shows its potential applications in knowledge acquisition, reasoning rule generation, and theorem finding, reports some current results of our experiments with EnCal, and suggests some important research problems.
\end{abstract}

\section{Keywords}

Entailment calculus, Forward deduction, Reasoning rule generation, Theorem finding

\section{INTRODUCTION}

Reasoning is the process of drawing new conclusions from some premises which are known facts and/or assumed hypothesis. A logically valid reasoning is a reasoning such that its process of drawing new conclusions from premises is justified based on some logical criterion in order to obtain correct conclusions. Therefore, a reasoning may be valid on a logical criterion but invalid on another. Automated reasoning is concerned with the execution of computer programs that assist in solving problems requiring reasoning. Knowledge Engineering (KE) is a discipline concerned with constructing and maintaining knowledge bases to store knowledge of various domains in the real world and using automated reasoning based on the knowledge to solve problems in the domains that ordinarily require human reasoning. Therefore, two key issues in $\mathrm{KE}$ are how to construct and maintain knowledge bases and how to automatically reasoning new and correct knowledge from known knowledge.

In recent years, a lot of knowledge-based systems have been developed and some of them have been used for solving problems in the real world. However, the current knowledge-based systems have a common inadequacy, i.e., they cannot reason about those situations and/or problems that have not been considered by their developers and/or users. A major cause of this inadequacy is that the systems cannot autonomously generate new and valid reasoning rules from those existing reasoning rules and facts that are programmed or inputted in the systems by their developers or users (Hayes-Roth, Waterman, and Lenat, 1983, Garcia and Chien, 1991). How can a knowledge-based system generate new and valid reasoning rules autonomously from the existing rules and facts in the system? This is an inevitable problem for making current knowledge-based systems more powerful and flexible.

On the other hand, although from 1950s many automated reasoning system for theorem proving have been developed and some difficult theorems have been automatically proved using the systems, at present there is no automated reasoning system can form some concept and/or 
find some theorem in a domain that are completely new and interesting to the scientists working on the domain (Simon, 1983, 1995). The problem of automated theorem finding, i.e., "what properties can be identified to permit an automated reasoning program to find new and interesting theorems, as opposed to proving conjectured theorems?" which was proposed by Wos 1988 as the thirty-first of 33 open research problems in automated reasoning, is still open until now (Wos, 1988, 1993). How can an automated reasoning system form new concept and/or find new theorem autonomously from known knowledge? In order to provide scientists with more powerful automatic tools assisting in scientific research and make computers serve as better partners of scientists, we have to answer this problem.

To solve the above two problems, it is indispensable to establish a domain-independent fundamental theory that underlies an autonomous reasoning mechanism and then develop automatic reasoning tools working based on the fundamental theory to support the autonomous reasoning mechanism.

Recently, the present author has proposed some paradox-free relevant logics (Cheng, 1996) and shown that an entailment calculus based on the paradox-free relevant logics can underlie reasoning rule generation in knowledge-based systems (Cheng, 1994) and automated theorem finding (Cheng, 1995). We are developing an automated forward deduction system for generalpurpose entailment calculus, named EnCal, which can support entailment calculus based on the paradox-free relevant logics as well as other logics. This paper presents the fundamental design ideas, working principles, and implementation of EnCal, shows its potential applications in knowledge acquisition, reasoning rule generation, and theorem finding, reports some current results of our experiments with EnCal, and suggests some important research problems.

\section{LOGICAL BASIS OF ENTAILMENT CALCULUS}

It is probably difficult, if not impossible, to find a sentence form in various natural and social scientific publications which is more generally used to describe various definitions, propositions, theorems, laws, and principles than the sentence form of "if ... then ... ." A sentence of the form "if ... then ..." is usually called a conditional which states that there exists a conditional and/or causal relationship between the "if" part and the "then" part of that sentence. Natural and social scientists always use conditionals in their descriptions of various definitions, propositions, theorems, laws and principles to connect a concept, fact, situation, or conclusion and its sufficient conditions. Indeed, a major work of almost all, if not all, scientists is to discover some conditional and/or causal relationships between various phenomena, data, and laws in their research fields.

In logic, the notion abstracted from various conditionals is called "entailment." In general, an entailment, for instance, "A entails B" or "if A then B," must concern two parts which are connected by connective "... entails ..." or "if ... then ..." and called the antecedent and the consequent of that entailment, respectively. The truth-value and/or validity of an entailment depends not only on the truth-values of its antecedent and consequent but also more essentially on a necessarily relevant and/or conditional relation between its antecedent and consequent. The notion of entailment plays the most essential role in human logical thinking because any reasoning must invoke it. Therefore, it is historically always the most important subject studied in logic and is regarded as the heart of logic (Anderson and Belnap Jr., 1975).

When we study and use logic, the notion of entailment may appear in both the object logic (i.e., the logic we are studying) and the meta-logic or observer's logic (i.e., the logic we are using to study the object logic). In object logic, there usually is a connective to represent the notion of entailment. On the other hand, in meta-logic, the notion of entailment is often used to represent a valid logical consequence relation. From the viewpoint of object logic, there are two kinds of entailments. One kind is empirical entailments and the other kind is logical entailments. For a logic, an entailment is called an empirical entailment of the logic if its truth-value, in the sense of that logic, depends on the contents of its antecedent and consequent (i.e., from the 
viewpoint of the logic, the relevant relation between the antecedent and the consequent of that entailment is regarded to be empirical); an entailment is called a logical entailment of the logic if its truth-value, in the sense of that logic, depends only on its abstract form but not on the contents of its antecedent and consequent, and therefore, it is considered to be universally true or false (i.e., from the viewpoint of the logic, the relevant relation between the antecedent and the consequent of that entailment is regarded to be logical). Indeed, the most intrinsic difference between some different logic systems is to regard what class of entailments as logical entailments.

Not all kinds of logic systems can serve well as the domain-independent fundamental theory to underlie an autonomous reasoning mechanism. There is an essential requirement for the logic system to be used as the fundamental theory, i.e., for any set of facts and entailments given as premises to be considered as true and/or valid, any entailment that is obtained as the conclusion of a deduction based on the logic system from the premises must be valid and must be new to the premises.

Because classical mathematical logic, where the notion of entailment is represented by the extensional truth-function material implication, accepts many implicational paradoxes as its logical theorems, it cannot satisfy the above requirement. All logic systems where the entailment is directly or indirectly represented by the material implication have the similar implicational paradox problem as that in classical mathematical logic. Although relevant logics such as system $\mathbf{T}$ of ticket entailment, system $\mathbf{E}$ of entailment, and system $\mathbf{R}$ of relevant implication are free of implicational paradoxes (Anderson and Belnap Jr., 1975, Anderson, Belnap Jr., and Dunn, 1992), they still accept conjunction-implicational paradoxes and disjunction-implicational paradoxes as logical theorems (Cheng, 1991). As a result, they also cannot satisfy the above requirement. Recently, the present author proposed some new paradox-free relevant logics $\mathbf{T c}, \mathbf{E c}$, and $\mathbf{R c}$ which are not only free of implicational paradoxes but also free of conjunction-implicational and disjunction-implicational paradoxes (Cheng, 1996), and therefore, they can satisfy the above requirement and serve well as the domainindependent fundamental theory to underlie an autonomous reasoning mechanism.

In general, both the logical theorems of a logic system and empirical theorems deduced based on the logic from some premises are an infinite set of formulas, even though the premises are finite. In order to develop a computational tool for reasoning about logical and empirical entailments, we have to find some method to limit the range of candidates for "new knowledge" to a finite set of formulas. The strategy the present author adopted is to sacrifice the completeness to get the finite set of candidates. This is based on the present author's conjecture that almost all "new knowledge" can be deduced from the premises by finite inference steps concerned with finite number of low degree logical entailments.

A formal logic system $\mathrm{L}$ is a triplet $\left(\mathrm{F}(\mathrm{L}), \mathrm{I}_{\mathrm{L}}, \mathrm{Th}(\mathrm{L})\right)$ where $\mathrm{F}(\mathrm{L})$ is the set of all well formed formulas of $\mathrm{L}, \mathrm{I}_{\mathrm{L}}$ is the logical consequence relation of $\mathrm{L}$ such that for $\mathrm{P} \subseteq \mathrm{F}(\mathrm{L})$ and $\mathrm{C} \in \mathrm{F}(\mathrm{L}), \mathrm{P}$ $\mathrm{I}_{\mathrm{L}} \mathrm{C}$ means that within the framework of $\mathrm{L}$ taking $\mathrm{P}$ as premises we can obtain $\mathrm{C}$ as a valid conclusion, and $\mathrm{Th}(\mathrm{L})$ is the set of logical theorems of $\mathrm{L}$ such that $\phi \mathrm{I}_{\mathrm{L}} \mathrm{t}$ holds for any $t \in T h(L)$. According to the representation of the logical consequence relation of a logic, the logic can be represented as a Hilbert style formal system, or a Gentzen natural deduction system, or a Gentzen sequent calculus system.

Let $\left(\mathrm{F}(\mathrm{L}), \mathrm{I}_{\mathrm{L}}, \mathrm{Th}(\mathrm{L})\right)$ be a formal logic system, $\mathrm{P} \subseteq \mathrm{F}(\mathrm{L})$ and $\mathrm{P} \neq \phi$. A formal theory with premises $P$ based on $L$, denoted by $\mathrm{T}_{\mathrm{L}}(\mathrm{P})$, is defined as follows:

$$
\mathrm{T}_{\mathrm{L}}(\mathrm{P})=_{\mathrm{df}} \mathrm{Th}(\mathrm{L}) \cup \mathrm{T}_{\mathrm{L}}{ }^{\mathrm{e}}(\mathrm{P}) \text { where } \mathrm{T}_{\mathrm{L}}{ }^{\mathrm{e}}(\mathrm{P})=_{\mathrm{df}}\left\{\mathrm{A}|\mathrm{P}|-_{\mathrm{L}} \mathrm{A} \text { and } \mathrm{A} \notin \mathrm{Th}(\mathrm{L})\right\}
$$

where $\mathrm{Th}(\mathrm{L})$ and $\mathrm{T}_{\mathrm{L}}{ }^{\mathrm{e}}(\mathrm{P})$ is called the logical part and the empirical part of the formal theory, respectively, and any element of $\mathrm{T}_{\mathrm{L}}^{\mathrm{e}}(\mathrm{P})$ is called an empirical theorem of the formal theory. 
An entailment calculus is a formal logical system where the notion of entailment is represented by a primitive connective and a part of its logical theorems are entailments.

For a formal logic system where the notion of entailment is represented by primitive connective " $\Rightarrow$ ", a formula is called a zero degree formula if and only if there is no occurrence of $\Rightarrow$ in it; a formula of the form $\mathrm{A} \Rightarrow \mathrm{B}$ is called a first degree formula (also called a first degree entailment) if and only if both $\mathrm{A}$ and $\mathrm{B}$ are zero degree formulas; a formula of the form $\neg \mathrm{A}$ is called a first degree formula if and only if $\mathrm{A}$ is a first degree formula; a formula of the form $\mathrm{A} * \mathrm{~B}$, where $*$ is conjunction or disjunction connective, is called a first degree formula if and only if both A and B are first degree formulas, or one of A and B is a first degree formula and the another is a zero degree formula. Let $k$ be a natural number. A formula of the form $A \Rightarrow B$ is called a $k^{\text {th }}$ degree formula (also called a $k^{\text {th }}$ degree entailment) if and only if both $\mathrm{A}$ and $\mathrm{B}$ are $k-1^{\text {th }}$ degree formulas, or one of $A$ and $B$ is a $k-1^{\text {th }}$ degree formula and the another is a $j^{\text {th }}(j<k-$ 1) degree formula; a formula of the form $\neg A$ is called a $k^{\text {th }}$ degree formula if and only if $A$ is a $\mathrm{k}^{\text {th }}$ degree formula; a formula of the form $\mathrm{A} * \mathrm{~B}$, where $*$ is conjunction or disjunction connective, is called $\mathrm{a}^{\text {th }}$ degree formula if and only if both $\mathrm{A}$ and $\mathrm{B}$ are $\mathrm{k}^{\text {th }}$ degree formulas, or one of $A$ and $B$ is a $k^{\text {th }}$ degree formula and the another is $a j^{\text {th }}(j<k)$ degree formula.

Let $\left(\mathrm{F}(\mathrm{L}), \mathrm{I}_{\mathrm{L}}, \mathrm{Th}(\mathrm{L})\right)$ be a formal logic system and $\mathrm{k}$ be a natural number. The $k^{\text {th }}$ degree fragment of $\mathrm{L}$, denoted by $\mathrm{Th}^{\mathrm{k}}(\mathrm{L})$, is a set of logical theorems of $\mathrm{L}$ which is inductively defined as follows (in the terms of Hilbert style formal system): (1) if $\mathrm{A}$ is an axiom of $\mathrm{L}$, then $A \in T^{k}(L),(2)$ if $A$ is a $j^{\text {th }}(j \leq k)$ degree formula which is the result of applying an inference rule of $L$ to some members of $\mathrm{Th}^{\mathrm{k}}(\mathrm{L})$, then $A \in \mathrm{Th}^{\mathrm{k}}(\mathrm{L})$, and (3) Nothing else are members of $\mathrm{Th}^{\mathrm{k}}(\mathrm{L})$, i.e., only those obtained from repeated applications of (1) and (2) are members of $\mathrm{Th}^{\mathrm{k}}(\mathrm{L})$. Obviously, the definition of the $\mathrm{k}^{\text {th }}$ degree fragment of logic $\mathrm{L}$ is constructive.

Note that the $\mathrm{k}^{\text {th }}$ degree fragment of logic $\mathrm{L}$ does not necessarily include all $\mathrm{k}^{\text {th }}$ degree logical theorems of $L$ because it is possible for $L$ that deductions of some $\mathrm{k}^{\text {th }}$ degree logical theorems of $\mathrm{L}$ must invoke those logical theorems whose degrees are higher than $\mathrm{k}$. On the other hand, the following holds obviously: $\operatorname{Th}^{0}(\mathrm{~L}) \subset \mathrm{Th}^{1}(\mathrm{~L}) \subset \ldots \mathrm{Th}^{\mathrm{k}-1}(\mathrm{~L}) \subset \mathrm{Th}^{\mathrm{k}}(\mathrm{L}) \subset \mathrm{Th}^{\mathrm{k}+1}(\mathrm{~L}) \subset \ldots$

Let $\left(F(L), I_{L}, T h(L)\right)$ be a formal logic system, $P \subset F(L)$, and $k$ and $j$ be two natural numbers. A formula $\mathrm{A}$ is said to be $j^{\text {th }}$-degree-deducible from $P$ based on $T h^{k}(L)$ if and only if there is an finite sequence of formulas $f_{1}, \ldots, f_{n}$ such that $f_{n}=A$ and for all $i(i \leq n)(1) f_{i} \in T h^{k}(L)$, or (2) $f_{i} \in P$, or (3) $f_{i}$ whose degree is not higher than $j$ is the result of applying an inference rule to some members $f_{j 1}, \ldots, f_{j m}(j 1, \ldots, j m<i)$ of the sequence. If $P \neq \phi$, then the set of all formulas which are $j^{\text {th }}$-degree-deducible from $\mathrm{P}$ based on $\operatorname{Th}^{\mathrm{k}}(\mathrm{L})$ is called the $j^{\text {th }}$ degree fragment of the formal theory with premises $P$ based on $\operatorname{Th}^{k}(L)$, denoted by $\mathrm{T}^{\mathrm{j}} \mathrm{Th}_{(\mathrm{L})}(\mathrm{P})$. A formula $\mathrm{A}$ is said to be $j^{\text {th }}$-degree-deductive from $P$ based on $T h^{k}(L)$ if and only if it is $j^{\text {th }}$-degree-deducible from $P$ based on $\mathrm{Th}^{\mathrm{k}}(\mathrm{L})$ but not $j-1^{\text {th }}$-degree-deducible from $P$ based on $\mathrm{Th}^{\mathrm{k}}(\mathrm{L})$.

The notion of $j^{\text {th }}$-degree-deductive can be used as a metric to measure the difficulty of deducing an empirical theorem from given premises $\mathrm{P}$ based on logic L. The difficulty is relative to the complexity of problem being investigated as well as the strength of underlying logic L.

Based on the above definitions, we have the following result.

Let $T_{R L}(P)$ be a formal theory where RL is $\mathbf{T c}$, or $\mathbf{E c}$, or $\mathbf{R c}$. If $P$ is finite, then $T^{j}{ }_{{ }_{h}} k_{(R L)}(P)$ must be finite. This is also true even if $\mathrm{T}^{\mathrm{j}} \mathrm{Th}^{\mathrm{k}}(\mathbf{R L})$ 


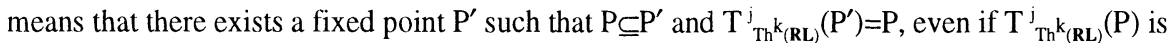
directly or indirectly inconsistent.

The above result about paradox-free relevant logics $\mathbf{T c}, \mathbf{E c}$, and $\mathbf{R c}$ does not hold for those paradoxical logics such as classical mathematical logic and its various extensions, and relevant logics $\mathbf{T}, \mathbf{E}$ and $\mathbf{R}$ because these logics accept implicational, conjunction-implicational, or disjunction-implicational paradoxes as logical theorems.

\section{EnCal AND ITS APPLICATIONS}

Probably, the most intrinsic difference between discovery and proof is that discovery has no explicitly defined target as its goal. Any discovery must be unknown before the discovery process is completed. Therefore, any scientific discovery process must include an epistemic process to gain knowledge of or to ascertain the existence of some empirical and/or logical entailments previously unknown or unrecognized. Since the epistemic process has no explicitly defined target, the sole criterion it must act according to is to reason correct conclusions when the premises are correct. It is logic that can underlie valid reasoning. On the other hand, any backward and/or refutation deduction system cannot serve as an autonomous reasoning mechanism to form and/or discover some completely new things. What we need is an autonomous forward reasoning system.

We are developing an automated forward deduction system for general-purpose entailment calculus, named EnCal, which provides its users with the following major facilities. For a logic $\mathrm{L}$ which may be a propositional logic, a first-order predicate logic, or a second-order predicate logic, a non-empty set $\mathrm{P}$ of formulas as premises, a natural number $\mathrm{k}$, and a natural number $\mathrm{j}$ all specified by the user, EnCal can

(1) reason out all logical theorem schemata of the $\mathrm{k}^{\text {th }}$ degree fragment of $\mathrm{L}$,

(2) verify whether or not a formula is a logical theorem of the $\mathrm{k}^{\text {th }}$ degree fragment of $\mathrm{L}$, if yes, then give the proof,

(3) reason out all empirical theorems of the $j^{\text {th }}$ degree fragment of the formal theory with premises $\mathrm{P}$ based on $\mathrm{Th}^{\mathrm{k}}(\mathrm{L})$, and

(4) verify whether or not a formula is an empirical theorem of the $j^{\text {th }}$ degree fragment of the formal theory with premises $\mathrm{P}$ based on $\operatorname{Th}^{\mathrm{k}}(\mathrm{L})$, if yes, then give the proof.

Although EnCal is designed and implemented for entailment calculi of relevant logics, it can also be used for entailment calculi of classical mathematical logic and its various extensions without problems in principle. However, because classical mathematical logic has an enormous number of paradoxes as its logical theorems, probably an entailment calculus of classical mathematical logic or its extension performed by EnCal has no practical applications.

EnCal consists of the following major parts all of which are implemented in Common LISP:

(1) EnCal-P: Briefly, EnCal-P is a pattern-driven implementation of the inference rule of Modus Ponens for propositional logics. It works with a pattern pool and a datum pool. For the given axiom schemata of a specified logic $\mathrm{L}$ and the degree $\mathrm{k}$, it puts all axiom schemata of $\mathrm{L}$ in the pattern and datum pools at first, and then repeatedly applies Modus Ponens to every element of the pattern pool and every element of the datum pool such that once a new logical theorem schema whose degree is not higher than $\mathrm{k}$ is reasoned out, it is added in both the pattern and datum pools. This process continues until no new logical theorem schema whose degree is not higher than $\mathrm{k}$ can be reasoned out.

(2) EnCal-Q: EnCal-Q is an extension of EnCal-P to deal with first order predicate logics. Its working principle is similar to that of EnCal-P but it has the ability to deal with individual quantifiers and variables. 
(3) EnCal-E: In contrast to that EnCal-P and EnCal-Q are tools for reasoning about logical entailments, EnCal-E is a tool for reasoning about empirical entailments with logical theorem schema generated by EnCal-P and EnCal-Q. It also works with a pattern pool and a datum pool. For the given kth degree fragment of a specified logic L, the premises, and the degree $\mathrm{m}$, it puts all logical theorem schemata of the kth degree fragment of $\mathrm{L}$ in the pattern tool and puts in all premises in datum pool at first, and then repeatedly applies the inference rule of Modus Ponens and other inference rules specified by the user to every element of the pattern pool and every element of the datum pool such that once a new empirical theorem whose degree is not higher than $\mathrm{m}$ is reasoned out, it is added in the datum pool. This process continues until no new empirical theorem whose degree is not higher than $\mathrm{m}$ can be reasoned out.

(4) EnCal-Q2: EnCal-Q2 is an extension of EnCal-Q to deal with second order predicate logics. Its working principle is similar to that of EnCal-Q but it has the ability to deal with predicate quantifiers and variables as well as individual quantifiers and variables.

(5) EnCal-E2: EnCal-E2 is an extension of EnCal-E to deal with second order theories.

(6) EnCal-T: EnCal-T is a tool kit for the user to edit input data for EnCal, transform the reasoning results into various forms specified by the user, and provide the user with various set operations on the reasoning results.

Within the framework of relevant logic, as an automated forward deduction system for general-purpose entailment calculus, EnCal has the following potential applications:

First, EnCal can be used as a domain-independent reasoning engine in knowledge-based systems for autonomous generation of reasoning rules. Taking those existing reasoning rules and facts that are programmed or inputted in the systems by their developers or users as premises, EnCal can autonomously generate some new reasoning rules which are valid in the sense of entailment if any of premises is valid and/or true.

Second, EnCal can be used as a domain-independent verification tool in knowledge-based systems for automatic verification of reasoning rules. For a given knowledge and/or rule base, EnCal can automatically verify whether or not a reasoning rule or fact acquired from a domain expert is valid and consistent, in the sense of entailment, with the knowledge and/or rule base.

Third, EnCal can be used as an incremental knowledge acquisition tool for domain experts. Taking knowledge that has been explicitly represented in formulas by a domain expert and/or knowledge engineer as premises, EnCal can automatically reason out some new knowledge which are valid in the sense of entailment if any of premises is valid and/or true.

Finally, EnCal can serve as an assistant of scientists for scientific discovery. For some formulas given as premises by a scientist, EnCal can automatically find some new theorems which are deducible from the premises and guarantee the validity of the theorems in the sense of entailment if any of premises is valid and/or true.

An important feature of EnCal is that it dose not require that the premises provided by the user must be consistent because it works based on paradox-free relevant logics. In general, a formal theory constructed as a purely deductive science (e.g., classical mathematical logic and its various extensions) is consistent. However, almost all, if not all, formal theories constructed based on empirical and/or experiential sciences are generally indirectly inconsistent. Therefore, EnCal can work well for empirical and/or experiential engineers and scientists as well as logicians and mathematicians.

\section{SOME CURRENT RESULTS OF EXPERIMENTS WITH EnCal}

It is a well-known fact that classical mathematical logic has a lot of implicational paradoxes as its logical theorems. But, how many paradoxes has the logic? Until recently, there is no quantitative answer for this question even if it is important in practices. Using EnCal, we did a 
quantitative comparison of logical theorem schemata of 1 st, 2 nd, and 3rd degree fragments of classical mathematical logic and relevant logics. A part of the results of the comparison is shown in Table 1, where $\mathbf{T}_{\Rightarrow}, \mathbf{E}_{\Rightarrow}, \mathbf{R}_{\Rightarrow}$, and $\mathbf{C M L}$, are the purely implicational fragments of relevant logics $\mathbf{T}, \mathbf{E}$, and $\mathbf{R}$, and classical mathematical logic $\mathbf{C M L}$, respectively; $\mathbf{T}_{\Rightarrow \rightarrow \neg}, \mathbf{E}_{\Rightarrow, \neg}$, $\mathbf{R}_{\Rightarrow, \neg}$, and $\mathbf{C M L}_{\rightarrow, \neg}$ are the implication-negation fragments of T, E, R, and CML, respectively; the numbers in the parentheses are the numbers of axiom schemata of the logics.

Table 1 A quantitative comparison of logical theorem schemata of 3rd degree fragment of various logics

\begin{tabular}{|c|c|c|c|c|c|c|c|c|c|}
\hline Logics & $\mathbf{T}_{\Rightarrow}$ & $\mathbf{E}_{\Rightarrow}$ & $\mathbf{R}_{\Rightarrow}$ & $\mathbf{C M L}$ & $\mathbf{T}_{\Rightarrow, \mathfrak{}}$ & $\mathbf{E}_{\Rightarrow, \mathfrak{r}}$ & $\mathbf{R}_{\Rightarrow, \neg}$ & $\mathbf{C M L}_{\rightarrow,-}$ & $\mathbf{T c}$ \\
\hline Theorem & 5 & 11 & 10 & 48 & 68 & 104 & 384 & $73115+$ & 11341 \\
schemata & $(5)$ & $(9)$ & $(7)$ & $(5)$ & $(8)$ & $(12)$ & $(10)$ & $(11)$ & $(14)$ \\
\hline
\end{tabular}

We found that an enormous number of logical theorems of the classical mathematical logic are not accepted by the relevant logics. This fact also tell us that the consideration to get nonparadoxical logical theorems by filtering paradoxes from the classical mathematical logic and/or its various extensions is not practical.

Since almost all mathematics can be formulated in the language of set theory, the set theory has been regarded as the ultimate proving ground for automated theorem proving programs (Boyer et al., 1986, Quaife, 1992). This should be also true in automated theorem finding. We take set theory as the starting point of our experiments on automated theorem finding with EnCal and are finding "new and interesting theorems" in NBG set theory by EnCal (Cheng, 1995). The underlying logic we adopted is Tcqe which is an extension of Tc such that it has quantifier and equality and relative axiom schemata. Using EnCal, we have obtained

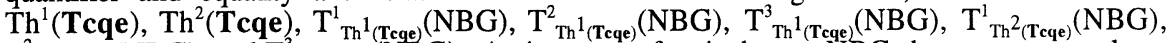
$\mathrm{T}^{2}{ }_{\mathrm{Th}}{ }^{2}(\mathrm{Tcye})(\mathrm{NBG})$, and $\mathrm{T}^{3}{ }_{\mathrm{Th}^{2}(\mathrm{Tcqe})}(\mathrm{NBG})$. An important fact is that no NBG theorem reasoned out by EnCal automatically is paradoxical. We are continuing the experiment using the 3rd degree fragment of Tcqe. We are also doing a comparison of the theorems reasoned out by us using EnCal automatically and the theorems proved by Quaife using OTTER automatically or semiautomatically (Quaife, 1992).

Other experiments we are doing using EnCal include automated theorem finding in Peano arithmetic and number theory, new reasoning rule generation in Lenat's AM system, and development of an autonomous information system to support theorem finding in mathematics.

\section{CONCLUDING REMARKS}

We have presented the logical basis of entailment calculus, an automated forward deduction system for general-purpose entailment calculus, named EnCal, and some current results of our experiments with EnCal. EnCal can be used as a domain-independent reasoning engine in various knowledge-based systems which require an autonomous reasoning mechanism to get new logical and empirical entailments.

There are many important and challenging research problems on this direction. For example, an issue which is both theoretically and practically important is that we need some kind of normal form to represent equivalence classes of formulas in a logic where the notion of entailment is represented by a primitive connective. In fact, almost all existing automated deduction methods and/or techniques convert formulas into some kind of normal form as a first step. Since these methods and/or techniques are developed based on the classical mathematical logic or its various extensions where conjunction and disjunction are used as primitive connectives, they usually convert formulas into conjunctive or disjunctive normal form. 
However, for a logic where the notion of entailment is represented by a primitive connective, conjunctive and disjunctive normal forms are obviously inadequate. We have to define an equivalence relation on formulas of the logic according to its model theory and then find a new kind of normal form to represent entailments. Another big issue, which is concerned with automated theorem finding, is that in order to make a program able to automatically discover some thing that is completely new to us, we have to tell the program what we have known. This requires us to construct a database which stores concepts, theorems, open problems of the special area in which we want to find some new thing using the program.

\section{REFERENCES}

Anderson, A. R. and Belnap Jr., N. D. (1975) Entailment: The Logic of Relevance and Necessity, Vol.I. Princeton University Press.

Anderson, A. R., Belnap Jr., N. D., and Dunn J. M. (1992) Entailment: The Logic of Relevance and Necessity, Vol.II. Princeton University Press.

Boyer, R., Lusk, E., McCune, W., Overbeek, R., Stickel, M., and Wos, L. (1986) Set Theory in First-Order Logic: Clauses for Godel's Axioms, Journal of Automated Reasoning, Vol.2, No.3, 287-327.

Cheng, J. (1991) Logical Tool of Knowledge Engineering: Using Entailment Logic rather than Mathematical Logic, in Proc. ACM 19th Annual Computer Science Conference, 228-238.

Cheng, J. (1994) Entailment Calculus as a Logical Tool for Reasoning Rule Generation and Verification, in Moving Toward Expert Systems Globally in the 21st Century (ed. J. Liebowitz) Cognizant Communication Co., 386-392.

Cheng, J. (1995) Entailment Calculus as the Logical Basis of Automated Theorem Finding in Scientific Discovery, in Systematic Methods of Scientific Discovery - Papers from 1995 Spring Symposium, Technical Report SS-95-03, AAAI Press, 105-110.

Cheng, J. (1996) The Fundamental Role of Entailment in Knowledge Representation and Reasoning, Journal of Computing and Information, Special Issue: Proc. 8th International Conference on Computing and Information.

Garcia, O. N. and Chien, Y.-T. (eds.) (1991) Knowledge-Based Systems: Fundamentals and Tools, IEEE-CS Press.

Hayes-Roth, F., Waterman, D. A., and Lenat D. B. (eds.) (1983) Building Expert Systems, Addison-Wesley.

Quaife, A. (1992) Automated Development of Fundamental Mathematical Theorems, Kluwer Academic.

Simon, H. A. (1983) Why Should Machines Learn? in Michalski, R. S., Carbonell, J. G., and Mitchell, T. M. (Eds.) Machine Learning: An Artificial Intelligence Approach, SpringerVerlag, 25-37.

Simon, H. A. (1995) What is a Systematic Method of Scientific Discovery? in Systematic Methods of Scientific Discovery - Papers from 1995 Spring Symposium, Technical Report SS-95-03, AAAI Press, 1-2.

Wos, L. (1988) Automated Reasoning: 33 Basic Research Problems, Prentice-Hall.

Wos, L. (1993) The Problem of Automated Theorem Finding, Journal of Automated Reasoning, Vol.10, No.1, 137-138.

\section{BIOGRAPHY}

Jingde Cheng received the B.Eng. degree in computer science from Tsinghua University, China in 1982, and M.Eng. and D.Eng. degrees in computer science from Kyushu University, Japan in 1986 and 1989, respectively. He is currently a professor of computer science and communication engineering at Kyushu University. His current research interests include logical basis of knowledge engineering, programming languages and systems, and knowledge-based software engineering. 